\title{
Unilateral versus bilateral iliac screws for spinopelvic fixation: are two screws better than one?
}

\author{
Rajiv Saigal, M.D., Ph.D., ${ }^{1}$ Darryl Lau, M.D., ${ }^{1}$ Rishi Wadhwa, M.D., ${ }^{1}$ Hai Le, B.S., ${ }^{2}$ \\ Morsi Khashan, M.D., ${ }^{3}$ Sigurd Berven, M.D., ${ }^{3}$ Dean Chou, M.D., ${ }^{1}$ \\ and Praveen V. Mummaneni, M.D. ${ }^{1}$
}

Departments of ${ }^{I}$ Neurological Surgery and ${ }^{3}$ Orthopedic Surgery, and ${ }^{2}$ School of Medicine, University of California, San Francisco, California

\begin{abstract}
Object. Long-segment spinal instrumentation ending at the sacrum places substantial biomechanical stress on sacral screws. Iliac (pelvic) screws relieve some of this stress by supplementing the caudal fixation. It remains an open question whether there is any clinically significant difference in sacropelvic fixation with bilateral versus unilateral iliac screws. The primary purpose of this study was to compare clinical and radiographic complications in the use of bilateral versus unilateral iliac screw fixation.

Methods. The authors retrospectively reviewed 102 consecutive spinal fixation cases that extended to the pelvis at a single institution (University of California, San Francisco) in the period from 2005 to 2012 performed by the senior authors. Charts were reviewed for the following complications: reoperation, L5-S1 pseudarthrosis, sacral insufficiency fracture, hardware prominence, iliac screw loosening, and infection. The t-test, Pearson chi-square test, and Fisher exact test were used to determine statistical significance.

Results. The mean follow-up was 31 months. Thirty cases were excluded: 12 for inadequate follow-up, 15 for lack of L5-S1 interbody fusion, and 3 for preoperative osteomyelitis. The mean age among the 72 remaining cases was 62 years (range 39-79 years). Forty-six patients underwent unilateral and 26 bilateral iliac screw fixation. Fortyone percent $(n=19)$ of the unilateral cases and $50 \%(n=13)$ of the bilateral cases were treated with reoperation $(p=$ $0.48)$. In addition, $13 \%(\mathrm{n}=6)$ of the unilateral and $19 \%(\mathrm{n}=5)$ of the bilateral cases developed L5-S1 pseudarthrosis $(\mathrm{p}=0.51)$. There were no sacral insufficiency fractures. Thirteen percent $(\mathrm{n}=6)$ of the unilateral and $7.7 \%(\mathrm{n}=2)$ of the bilateral cases developed postoperative infection $(\mathrm{p}=0.70)$.

Conclusions. In a retrospective single-institution study, single versus dual pelvic screws led to comparable rates of reoperation, iliac screw removal, postoperative infection, pseudarthrosis, and sacral insufficiency fractures. For spinopelvic fixation, placing bilateral (vs unilateral) pelvic screws produced no added clinical benefit in most cases. (http://thejns.org/doi/abs/10.3171/2014.3.FOCUS1428)
\end{abstract}

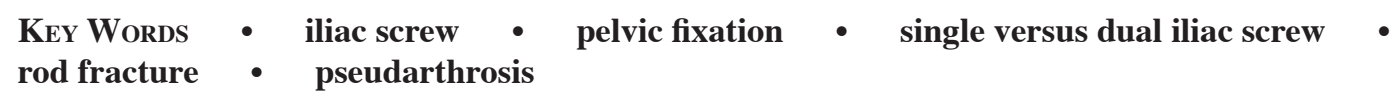

$\mathrm{L}$ ONG-Segment instrumented spinal fixation extending from L-2 or higher to the sacrum for adult spinal deformity places substantial biomechanical strain on the S-1 pedicle screws, especially during flexion. Strain on the S-1 screws may result in complications such as screw pullout or pseudarthrosis at the lumbosacral junction for these long-segment cases. To overcome this problem, many surgeons routinely use iliac (pelvic) screw fixation to supplement the sacral pedicle screws in long-segment fixation that extends to the sacrum. $2,3,5,11,12,15$ The addition of iliac screws decreases lumbosacral screw strain, ${ }^{6,8-10}$ which may lower the incidence of screw pullout, L5-S1 pseudarthrosis, and sacral insufficiency fracture at S1-2. Iliac fixation screws serve as temporary scaffolding to allow for the maturation of bony fusion across the lumbosacral junction. 7,14

The iliac screw fixation technique classically involves screw placement on both sides of the pelvis. Theoretical-

\footnotetext{
Abbreviations used in this paper: $\mathrm{BMP}=$ bone morphogenetic protein; PSIS = posterior superior iliac spine; UIV = uppermost instrumented vertebra.
}

ly, the bilateral iliac screw placement was thought to offer greater sacropelvic stability and construct strength than unilateral fixation. ${ }^{6,8-10}$ However, more recent biomechanical studies have indicated that unilateral and bilateral iliac screw fixation provide comparable construct biomechanics, that is, construct stiffness and lumbosacral range of motion. ${ }^{13}$ Some studies have even suggested that unilateral iliac screw fixation provides better clinical outcomes (for example, improved sagittal balance and reduced distal implant complications) than bilateral screw fixation. ${ }^{1}$ Other potential problems with bilateral fixation include prolonged operative time and increased risk for implant prominence..$^{13}$ Today, most spine deformity surgeons incorporate iliac screw fixation in fusion constructs extending from L-2 or higher to the sacrum, but the decision to place iliac screws unilaterally or bilaterally remains multifactorial and is often determined by surgeon preference.

Few studies have directly compared the clinical and radiographic outcomes of unilateral versus bilateral iliac screw fixation. The primary purpose of our study was to reveal any clinical or radiographic complications in the use of bilateral versus unilateral iliac screw fixation. 


\section{R. Saigal et al.}

\section{Methods}

This retrospective study was approved by the University of California, San Francisco, Committee on Human Research.

\section{Study Cohort}

We identified all adults in the period from 2005 to 2012 who had undergone posterior spinopelvic fixation with unilateral or bilateral iliac screw(s) placement by one of three spine surgeons (P.V.M., S.B., and D.C.). Patients with less than 6 months of postoperative imaging and followup evaluation were excluded from the final analysis. Other exclusionary criteria consisted of preoperative osteomyelitis and the lack of L5-S1 interbody fusion. Indications for pelvic screw placement varied (Table 1). The decision to use unilateral or bilateral iliac screws was based on surgeon preference. Since 2011, one senior author has tended to use unilateral iliac screws. The other senior author chose unilateral screws in settings of good S-1 fixation, uppermost instrumented vertebra (UIV) T-10 or lower, L5-S1 interbody cage placement, and minimal sagittal plane correction. He chose bilateral screws in cases of poor S-1 fixation, upper thoracic spine fixation, no L5-S1 interbody cage placement, significant sagittal plane correction, and/ or significant coronal plane correction.

\section{Data Collection}

A retrospective review and collection of patient demographics, operative details, and outcomes were done with the use of the electronic medical records. Data points included age, sex, specific surgical procedure, number of levels fused, spinal level, use of bone morphogenetic protein (BMP), use of L5-S1 interbody cage, and number of iliac screws implanted (bilateral or unilateral).

The primary outcomes of interest were perioperative and long-term complications. The definition of a complication in this study was an unforeseen event causing clinical symptom(s) and/or requiring additional medical or surgical intervention. Complications of specific interest included the need for reoperation, L5-S1 pseudarthrosis, iliac screw removal, sacral insufficiency/fracture, iliac screw loosening/fracture, wound/hardware infection, and hardware prominence. A neurosurgeon and an independent neuroradiologist evaluated all radiological findings. All patients underwent standardized 36-in longcassette radiographs preoperatively and at the last followup. Iliac screw loosening was counted if mentioned in the official radiology read of a CT or radiograph (or in any neurosurgery attending note after surgery), regardless of whether the patient was symptomatic. Any fracture of the iliac screws also counted toward iliac screw "loosening."

\section{TABLE 1: Indications for extension of lumbosacral fusions to the} ilium*

Grade II or higher L5-S1 spondylolisthesis

long-segment fusions to the sacrum (L-2 or above to S-1) treatment of L5-S1 pseudarthrosis

\footnotetext{
* Modified with permission from Tumialán and Mummaneni: Neurosurgery 63 (3 Suppl):183-190, 2008.
}

Hardware prominence was assessed through clinical history and physical examination at the time of follow-up.

\section{Iliac Screw Placement and Spinopelvic Fixation}

A Galveston-style iliac screw fixation technique was used for spinopelvic fixation. Iliac screw placement was done in a standard fashion as previously described by Tumialán and Mummaneni (Table 2), ${ }^{7,11,12}$ The iliac crest is first exposed by mobilizing the paraspinal muscles. After adequate exposure of the iliac crest is achieved, the posterior superior iliac spine (PSIS) is palpated. The subcutaneous tissue is dissected off the lumbosacral fascia toward the PSIS. An incision is made along the fascia overlying the PSIS. A bur is used to decorticate the bony cortex for an entry point, as described by Tumialán and Mummaneni..$^{15}$ A gearshift probe is introduced under fluoroscopy to create a pilot hole by aiming toward the anterior superior iliac spine. The screw path is palpated with a ballpoint probe to verify that neither the medial nor the lateral iliac crest cortex has been breached. Screw length is then measured, the screw path is tapped, and a screw with an appropriate length is inserted. The same technique is used on the contralateral side if the patient is undergoing bilateral iliac screw fixation, and the iliac screws are then connected to the longitudinal rods. Typical screw lengths are $65-80 \mathrm{~mm}$.

\section{Statistical Analysis}

The aim in this study was to determine whether there was a difference in complications between unilateral and bilateral iliac screw fixation. Therefore, patients were stratified into two treatment groups: unilateral screw fixation and bilateral screw fixation. Comparisons between the two groups were performed with descriptive statistics and univariate comparison tests. For categorical variables, the Pearson chi-square test was used when all values in the $2 \times 2$ contingency table were $\geq 10$; the Fisher exact test was substituted in contingency tables with any values $<10$. For continuous variables, a 2 -tailed Student t-test was used. A p value $<0.05$ was the threshold of statistical significance. All statistical analyses were performed with SPSS 21 (SPSS Inc.).

\section{Results}

Among 102 consecutive spinal fixation cases, 30 met the exclusionary criteria and were not included in the final analysis. Twelve cases were excluded for inadequate follow-up, another 15 lacked an L5-S1 interbody fusion, and 3 were excluded for preoperative osteomyelitis. Of the 72 remaining cases, 46 were treated with unilateral (Fig. 1) and 26 with bilateral (Fig. 2) iliac screw fixation. The mean age was 62 years for both groups (range 39-77 years for unilateral and 41-79 years for bilateral iliac screws, $p=0.95)$. In the unilateral iliac screw cohort, $76 \%(\mathrm{n}=35)$ of the patients were women; in the bilateral cohort, $77 \%(\mathrm{n}=20)$ were women $(\mathrm{p}=1.0)$.

Intraoperative instrumentation was statistically similar in both cohorts. The mean number of instrumented levels was 8 (range 5-16 levels) in the unilateral group and 9 (range 5-15 levels) in the bilateral group ( $\mathrm{p}=0.47$; 


\section{Unilateral versus bilateral iliac screws for spinopelvic fixation}

TABLE 2: Steps in pelvic screw fixation technique*

1. Expose the PSIS

2. Locate entry point of pelvic screw (iliac screw)

A. $1 \mathrm{~cm}$ deep to superficial edge of PSIS

B. $1 \mathrm{~cm}$ proximal to palpated inferior end of PSIS

3. Decorticate entry point $w /$ drill or awl

4. Use gearshift probe to create pilot hole for screw

A. aim for thick bone just above greater sciatic notch

i. typically $30^{\circ}-45^{\circ}$ down angle in axial plane $\& 30^{\circ}-45^{\circ}$ inferior in coronal plane

a. typical depth is $60-80 \mathrm{~mm}$

b. may use anteroposterior, pelvic inlet, \& obturator fluoroscopic views to guide trajectory

c. may expose superficial surface of ilium to guide screw trajectory in axial plane

5. Tap pilot hole (undersize tap)

6. Probe for cortical wall violation

7. Place pelvic screw

A. recess head into PSIS

8. Connect to lumbosacral rod inferior to S-1 level A. may use connector

* Modified with permission from Tumialán and Mummaneni: Neurosurgery 63 (3 Suppl):183-190, 2008.

Table 3). Seventy-two percent of patients in the unilateral group and $92 \%$ of those in the bilateral group received instrumentation extending up to the thoracic spine $(\mathrm{p}=$ $0.07)$. Sixty-one percent $(\mathrm{n}=28)$ of the unilateral and $50 \%(\mathrm{n}=13)$ of the bilateral cases involved the use of BMP to promote fusion ( $\mathrm{p}=0.37$ ). Pursuant to the study inclusion criteria, all cases involved the use of an L5-S1 interbody cage. There was no significant difference in any of the baseline data between the two cohorts.

Similarly, there was no significant difference between single and dual screws for key outcome complications (Table 4). Nearly the same number of patients required reoperation ( $41 \%$ unilateral cohort vs $50 \%$ bilateral cohort, $\mathrm{p}=0.48)$. The iliac screw was removed in a subsequent reoperation in $10.9 \%$ unilateral versus $11.5 \%$ bilateral screw cases $(p=1.0)$. Thirteen percent $(n=6)$ of the unilateral screw cases later demonstrated L5-S1 pseudarthrosis, as compared with $19 \%(\mathrm{n}=5)$ of the bilateral iliac screw cases $(\mathrm{p}=0.51)$. There were no sacral insufficiency fractures in either group.

Hardware prominence eventually developed in 1 $(2.2 \%)$ of the single iliac screw cases and $3(11.5 \%)$ of the bilateral iliac screw cases $(\mathrm{p}=0.13)$. Iliac screw loosening was seen radiographically in $26 \%(\mathrm{n}=12)$ of the unilateral iliac screw cases and $27 \%(n=7)$ of the bilateral iliac screw cases $(p=1.0)$. There was no significant difference in postoperative infection rates between the two groups: $13 \%(n=6)$ of unilateral vs $7.7 \%(n=2)$ of the bilateral iliac screw cases $(p=0.70)$. These data are shown in Table 4 and Fig. 3.
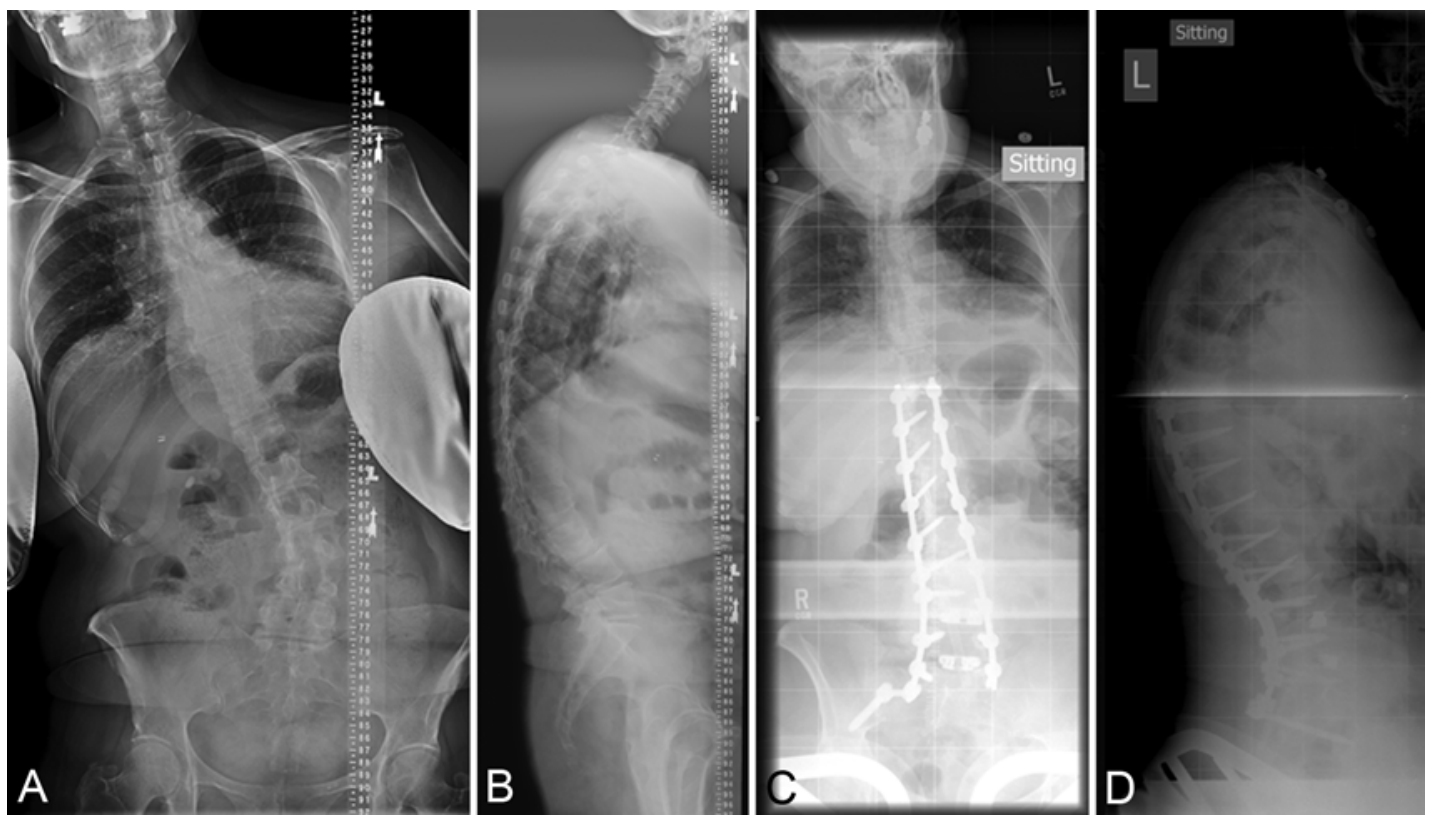

FIG. 1. Preoperative anteroposterior (A) and lateral (B) standing 36-in radiographs obtained in an adult with spinal deformity. Postoperative anteroposterior (C) and lateral (D) radiographs showing T10-pelvis fusion with unilateral iliac screw fixation. 


\section{R. Saigal et al.}
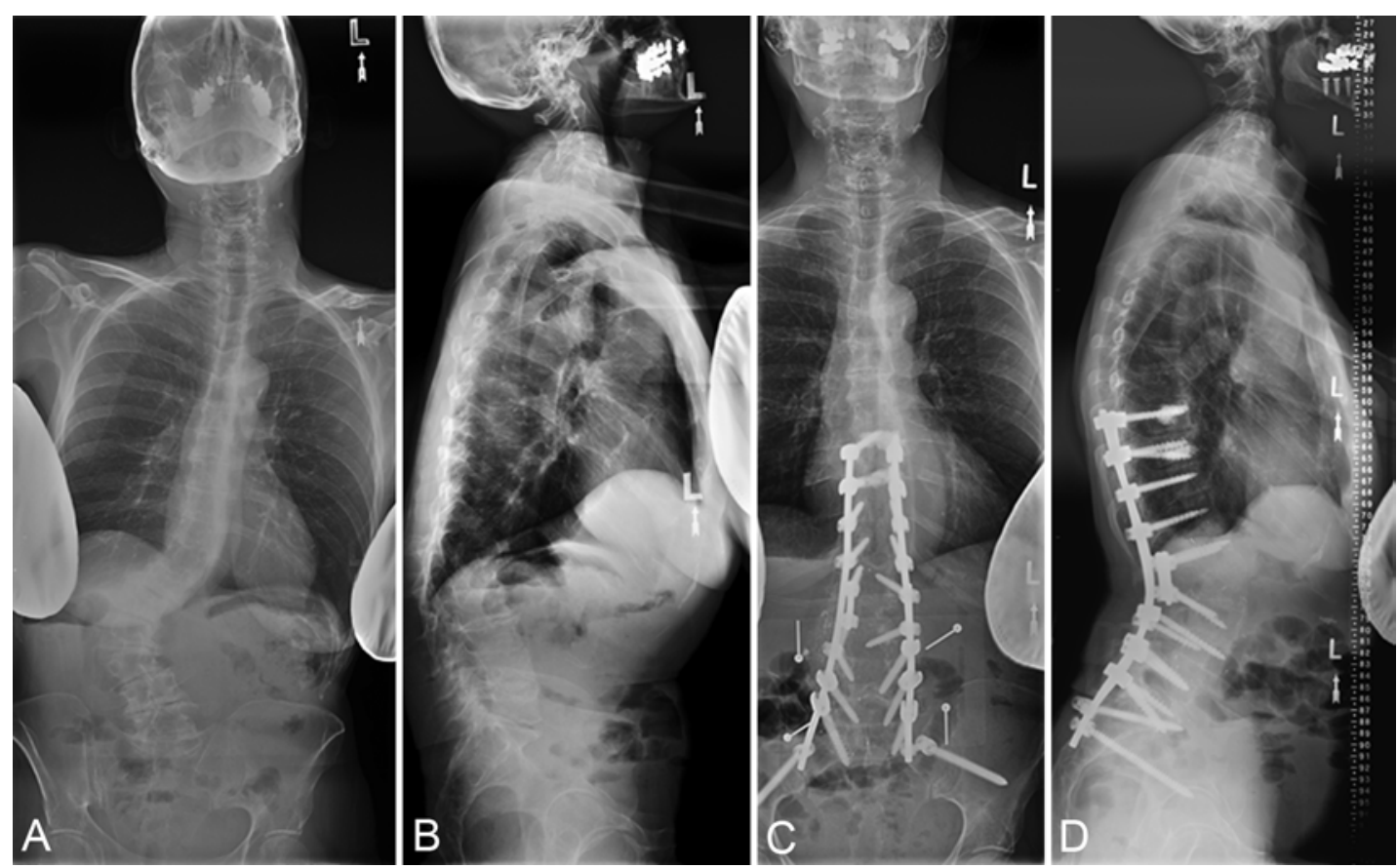

FIG. 2. Preoperative anteroposterior (A) and lateral (B) standing 36 -in radiographs obtained in an adult with spinal deformity. Postoperative anteroposterior (C) and lateral (D) radiographs showing long-segment thoraco-pelvis fusion with bilateral iliac screw fixation.

To ensure that shorter-segment fixations did not alter the results, we performed a post hoc subgroup analysis of only those cases above the thoracolumbar junction, that is, UIV of T-11 or above (31 unilateral cases and 20 bilateral cases; Table 5). There continued to be no statistical difference in postoperative complications data between the two cohorts. None of the differences approached significance, and the lowest $p$ value was 0.29 for L5-S1 pseudarthrosis $(13 \%[\mathrm{n}=4]$ unilateral vs $25 \%[\mathrm{n}=5]$ bilateral).

\section{Discussion}

Iliac screw fixation is often used to add biomechanical strength to long-segment fixation for adult spinal deformity cases. Considerable data suggest that lumbosacral screw strain is reduced by the addition of instrumentation to the pelvis, ${ }^{6,8-10}$ but what is less clear is whether one or two iliac screws is optimal for augmenting long-segment fusions. The potential upside of dual screw fixation would be greater biomechanical strength with decreased instrumentation-related postoperative complications such as screw pullout, pseudarthrosis, and sacral insufficiency fracture. ${ }^{16,17}$ The potential downside of a second screw is added muscle and fascia dissection, increased postoperative pain, problems with sexual function, ${ }^{2,4}$ increased cost, and possibly increased infection risk due to the added exposure and hardware.

In a retrospective study of consecutive cases at the University of California, San Francisco, in the period from 2005 to 2012, we compared single versus dual iliac screw fixation in terms of the rates of key postoperative complications. The two cohorts were generally well matched, with no significant difference in age, sex, instrumentation to the thoracic spine, number of instrumentation levels, use of BMP, and months of imaging follow-up. Prior to applying the study exclusion criteria, most cases did involve the use of an L5-S1 interbody cage, but some did not. The lack of cage placement could be a major confounder, independently negatively affecting pseudarthrosis rates. Therefore, we excluded 15 cases without L5-S1 interbody cages to produce more homogeneous and standardized groups for comparing unilateral and bilateral iliac screw fixation.

TABLE 3: Baseline demographics of single versus dual iliac screw fixation cohorts

\begin{tabular}{lccc}
\hline \multicolumn{1}{c}{ Parameter } & Unilat lliac Screw & Bilat lliac Screws & p Value \\
\hline total no. of patients & 46 & 26 & - \\
mean age in yrs (range) & $62(39-77)$ & $62(41-79)$ & 0.95 \\
no. of females (\%) & $35(76)$ & $20(77)$ & 1.0 \\
no. cases w/ instrumentation up to thoracic spine (\%) & $33(72)$ & $24(92)$ & 0.07 \\
mean no. of instrumented levels (range) & $8(5-16)$ & $9(5-15)$ & 0.47 \\
no. cases w/ BMP use (\%) & $28(61)$ & $13(50)$ & 0.37 \\
mean mos of imaging follow-up & 29 & 33 & 0.49 \\
\hline
\end{tabular}


Unilateral versus bilateral iliac screws for spinopelvic fixation

TABLE 4: Summary of overall complications

\begin{tabular}{lccc}
\hline & \multicolumn{3}{c}{ No. (\%) } \\
\cline { 2 - 3 } Complication & Unilat lliac Screw & Bilat lliac Screws & p Value \\
\hline reop & $19(41)$ & $13(50)$ & 0.48 \\
iliac screw removal & $5(10.9)$ & $2(11.5)$ & 1.0 \\
L5-S1 pseudarthrosis & $6(13)$ & $5(19)$ & 0.51 \\
sacral insufficiency fracture & $0(0)$ & $0(0)$ & 1.0 \\
hardware prominence & $1(2.2)$ & $3(11.5)$ & 0.13 \\
iliac screw loosening & $12(26)$ & $7(27)$ & 1.0 \\
infection & $6(13)$ & $2(7.7)$ & 0.70 \\
\hline
\end{tabular}

Although the difference was not statistically significant, it is worth noting that patients who underwent bilateral iliac screw fixation more frequently had spinal constructs that extended to the thoracic spine ( 24 of 26 cases vs 33 of 46 unilateral cases). Part of this difference is probably attributable to the surgeon's preference mentioned above. However, there was no statistical difference in the number of instrumented levels between the two groups (mean 8 for unilateral vs 9 for bilateral cohort, $p$ $=0.47$ ). Given the possibility that any added benefit from bilateral iliac screw fixation might only be seen with long-segment constructs, we performed a post hoc subgroup analysis for patients who underwent fusion with an UIV at T-11 or above. Neither the full cohort nor the subgroup analysis of longer-segment fusions (UIV T-11 or above) showed any significant difference between the two iliac fixation techniques. In the long-segment subgroup, there was no significant difference between the two techniques for rates of reoperation, need for iliac screw removal, infection, pseudarthrosis, hardware prominence, screw pullout, or sacral insufficiency fracture. For these key outcomes, the smallest p value was 0.29 for L5-S1 pseudarthrosis $(13 \%[\mathrm{n}=4$ ] for unilateral vs $25 \%[\mathrm{n}=5]$ for bilateral).

According to our data, there was no added benefit with the addition of bilateral iliac screw fixation over unilateral iliac fixation in the majority of cases. There did not appear to be any statistically significant harm in placing a contralateral iliac screw. In the full cohort of patients, bilateral fixation cases had higher rates of symptomatic hardware prominence (11.5\% vs 2.2\%) and higher levels of L5-S1 pseudarthrosis (19\% vs 13\%), but neither difference approached significance ( $\mathrm{p}=0.13$ and 0.51 , respectively).

In an era of increasing focus on value in medical practice, it is highly important to know whether added instrumentation improves outcome. The data here suggest that a second contralateral iliac screw does not. However, this conclusion comes with the usual qualifications of a retrospective single-center study, namely the possibility for sampling and selection bias. A larger, randomized, prospective multicenter study is needed to answer the question definitively.

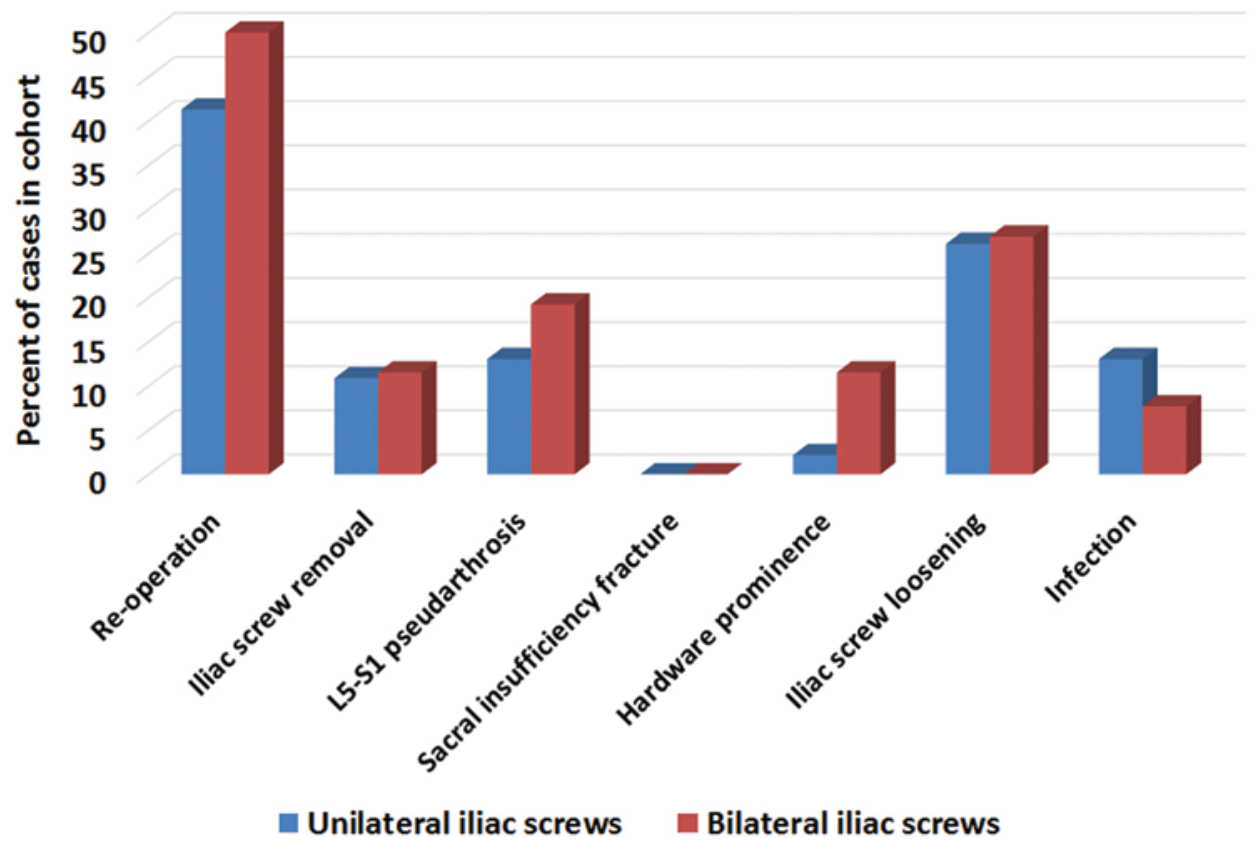

FIG. 3. Bar graph showing all primary outcome data. 
TABLE 5: Summary of complications in subgroup analysis (UIV T-11 or higher)

\begin{tabular}{lccc}
\hline & \multicolumn{3}{c}{ No. (\%) } \\
\cline { 2 - 3 } Complication & Unilat lliac Screw & Bilat lliac Screws & p Value \\
\hline reop & $16(52)$ & $10(50)$ & 0.91 \\
iliac screw removal & $5(16)$ & $2(10)$ & 0.69 \\
L5-S1 pseudarthrosis & $4(13)$ & $5(25)$ & 0.29 \\
sacral insufficiency fracture & $0(0)$ & $0(0)$ & 1.0 \\
hardware prominence & $1(3)$ & $2(10)$ & 0.55 \\
iliac screw loosening & $10(32)$ & $5(25)$ & 0.76 \\
infection & $5(16)$ & $2(10)$ & 0.69 \\
\hline
\end{tabular}

\section{Conclusions}

In a single-institution retrospective study, single versus dual pelvic screws led to comparable rates of reoperation, iliac screw removal, postoperative infection, pseudarthrosis, and sacral insufficiency fractures. For spinopelvic fixation, there may be no added benefit to placing bilateral (vs unilateral) pelvic screws in the majority of cases.

\section{Disclosure}

The authors report no conflict of interest concerning the materials or methods used in this study or the findings specified in this paper. Dr. Chou is a consultant for Globus, Orthofix, DePuy, and Medtronic. Dr. Mummaneni has ownership in and receives royalties from Thieme Publishing; has direct stock ownership in Spinicity; receives honoraria from Globus; receives honoraria and royalties from DePuy Spine; and receives royalties from QMP Publishing. Dr. Berven has ownership in Co-Align, Simpirica, and Providence Medical and is a consultant for Medtronic, Stryker, Globus, and Biomet.

Author contributions to the study and manuscript preparation include the following. Conception and design: Saigal, Mummaneni. Acquisition of data: Saigal, Lau, Wadhwa, Le, Khashan. Analysis and interpretation of data: Saigal, Lau, Wadhwa, Khashan. Drafting the article: Saigal, Lau, Wadhwa, Le. Critically revising the article: Saigal, Lau, Wadhwa, Mummaneni. Reviewed submitted version of manuscript: Saigal, Lau, Wadhwa, Berven, Chou, Mummaneni. Statistical analysis: Saigal. Administrative/technical/material support: Saigal, Mummaneni. Study supervision: Chou, Mummaneni.

\section{References}

1. Breakwell LM, Akbarnia BA, Boachie-Adjei O, Burton D, Ishikawa M, Canale SK, et al: Complications and 2 to 10 year results of long fusions to the sacrum and pelvis for adult deformity: is bilateral iliac fixation necessary?, in Programs and Abstracts of the 40th Annual Meeting of the Scoliosis Research Society, Miami, Florida, 2005. Milwaukee: Scoliosis Research Society, 2005, p 52 (Abstract)

2. Cho W, Mason JR, Smith JS, Shimer AL, Wilson AS, Shaffrey CI, et al: Failure of lumbopelvic fixation after long construct fusions in patients with adult spinal deformity: clinical and radiographic risk factors. Clinical article. J Neurosurg Spine 19:445-453, 2013

3. Fu KM, Smith JS, Burton DC, Kebaish KM, Shaffrey CI, Schwab F, et al: Revision extension to the pelvis versus primary spinopelvic instrumentation in adult deformity: comparison of clinical outcomes and complications. World Neurosurg [epub ahead of print], 2013

4. Hamilton DK, Smith JS, Nguyen T, Arlet V, Kasliwal MK, Shaffrey CI: Sexual function in older adults following thoracolumbar to pelvic instrumentation for spinal deformity. Clinical article. J Neurosurg Spine 19:95-100, 2013
5. Kostuik JP, Hall BB: Spinal fusions to the sacrum in adults with scoliosis. Spine (Phila Pa 1976) 8:489-500, 1983

6. Kostuik JP, Valdevit A, Chang HG, Kanzaki K: Biomechanical testing of the lumbosacral spine. Spine (Phila Pa 1976) 23:1721-1728, 1998

7. Kuklo TR, Bridwell KH, Lewis SJ, Baldus C, Blanke K, Iffrig TM, et al: Minimum 2-year analysis of sacropelvic fixation and L5-S1 fusion using S1 and iliac screws. Spine (Phila Pa 1976) 26:1976-1983, 2001

8. Lebwohl NH, Cunningham BW, Dmitriev A, Shimamoto N, Gooch L, Devlin V, et al: Biomechanical comparison of lumbosacral fixation techniques in a calf spine model. Spine (Phila Pa 1976) 27:2312-2320, 2002

9. Lee CK, Langrana NA: Lumbosacral spinal fusion. A biomechanical study. Spine (Phila Pa 1976) 9:574-581, 1984

10. McCord DH, Cunningham BW, Shono Y, Myers JJ, McAfee PC: Biomechanical analysis of lumbosacral fixation. Spine (Phila Pa 1976) 17 (8 Suppl):S235-S243, 1992

11. Moshirfar A, Rand FF, Sponseller PD, Parazin SJ, Khanna AJ, Kebaish KM, et al: Pelvic fixation in spine surgery. Historical overview, indications, biomechanical relevance, and current techniques. J Bone Joint Surg Am 87 (Suppl 2):89-106, 2005

12. Shen FH, Mason JR, Shimer AL, Arlet VM: Pelvic fixation for adult scoliosis. Eur Spine J 22 (Suppl 2):S265-S275, 2013

13. Tomlinson T, Chen J, Upasani V, Mahar A: Unilateral and bilateral sacropelvic fixation result in similar construct biomechanics. Spine (Phila Pa 1976) 33:2127-2133, 2008

14. Tsuchiya K, Bridwell KH, Kuklo TR, Lenke LG, Baldus C: Minimum 5-year analysis of L5-S1 fusion using sacropelvic fixation (bilateral S1 and iliac screws) for spinal deformity. Spine (Phila Pa 1976) 31:303-308, 2006

15. Tumialán LM, Mummaneni PV: Long-segment spinal fixation using pelvic screws. Neurosurgery 63 (3 Suppl):183-190, 2008

16. Wang MY, Ludwig SC, Anderson DG, Mummaneni PV: Percutaneous iliac screw placement: description of a new minimally invasive technique. Neurosurg Focus 25(2):E17, 2008

17. Wang MY, Williams S, Mummaneni PV, Sherman JD: Minimally invasive percutaneous iliac screws: initial 24 case experience with CT confirmation. J Spinal Disord Tech [epub ahead of print], 2012

Manuscript submitted January 16, 2014.

Accepted March 20, 2014.

Please include this information when citing this paper: DOI: 10.3171/2014.3.FOCUS1428.

Address correspondence to: Rajiv Saigal, M.D., Ph.D., University of California, San Francisco, Department of Neurological Surgery, 505 Parnassus Ave., Box 0112, San Francisco, CA 94143-0112. email: saigalr@neurosurg.ucsf.edu. 